\title{
Progressive Multiple Cranial Nerve Palsies as the Presenting Symptom of Meningeal Carcinomatosis from Occult Colon Adenocarcinoma
}

\author{
Yuji Kato ${ }^{1}$, Hidetaka Takeda ${ }^{1,2}$, Tomohisa Dembo ${ }^{1}$, Takuya Fukuoka ${ }^{1}$ and Norio Tanahashi ${ }^{1}$
}

\begin{abstract}
We herein describe a rare case of meningeal carcinomatosis in a 77-year-old woman who had bilateral deafness as the initial symptom of a previously undetected colon cancer malignancy. Meningeal carcinomatosis should be considered in the differential diagnosis in cases of abrupt-onset sensorineural deafness. Both MRI scans and cerebrospinal fluid evaluation are necessary diagnostic tools, and should be used in conjunction as each of these procedures could have false-negative results. This should apply even when there is no known primary malignancy.
\end{abstract}

Key words: meningeal carcinomatosis, multiple cranial nerve palsies, colon adenocarcinoma, deafness

(Intern Med 51: 795-797, 2012)

(DOI: 10.2169/internalmedicine.51.6682)

\section{Introduction}

Meningeal carcinomatosis (MC) is the malignant infiltration of the meninges from a distant solid tumor, most commonly adenocarcinoma of the breast or lung and melanoma $(1,2)$. MC also has been described infrequently in association with colon cancer (3). The majority of MC is diagnosed in patients with a known malignancy, and it is rare for a cranial nerve symptom to be the first manifestation. We present here an unusual case of MC caused by infiltration of the meninges by occult colon cancer and marked by progressive multiple cranial nerve palsies starting from bilateral sensorineural deafness.

\section{Case Report}

A 77-year-old woman presented with bilateral deafness and vertigo. Two weeks prior to admission, right-sided deafness developed over a few days and within one week this was followed by hearing impairment in the left ear. She developed progressive walking imbalance and right facial palsy and was admitted to a nearby medical institution. She had no significant prior medical history except for cataract. Although brain computed tomography (CT) was unremarkable, the patient's neurological condition continued to deteriorate with dysarthria and dysphagia. She was transferred to our hospital for further differential diagnosis and treatment.

On examination, she was well oriented and did not complain of headache. She had developed a persistent audible wheezing due to difficulty expectorating, and continued to experience peripheral facial palsy on the right side and bilateral sensorineural deafness.

Because of the progressive multiple cranial nerve palsies (VII, VIII, IX, X), a lumbar puncture was performed; cerebrospinal fluid (CSF) examination revealed mild pleocytosis (15 mononuclear and 9 polynuclear cells), mild elevation of protein levels $(68 \mathrm{mg} / \mathrm{dL}$; normal: $10-40 \mathrm{mg} / \mathrm{dL})$, and normal sugar levels. Polymerase chain reaction (PCR) of the CSF was negative for tuberculosis; bacterial cultures did not reveal any organisms. Magnetic resonance imaging (MRI) with gadolinium contrast revealed enhancement of the cerebellum and cranial nerves in the internal acoustic meatus (Fig. 1) Repeat CSF cytology showed evidence of degenerate atypical cells with features of metastatic adenocarcinoma (Fig. 2). These findings led to the diagnosis of MC, and the

${ }^{1}$ Department of Neurology and Cerebrovascular Medicine, Saitama International Medical Center, Saitama Medical University, Japan and ${ }^{2}$ Department of Neurology, International University of Health and Welfare, Japan

Received for publication October 6, 2011; Accepted for publication December 4, 2011

Correspondence to Dr. Yuji Kato, yujik@saitama-med.ac.jp 

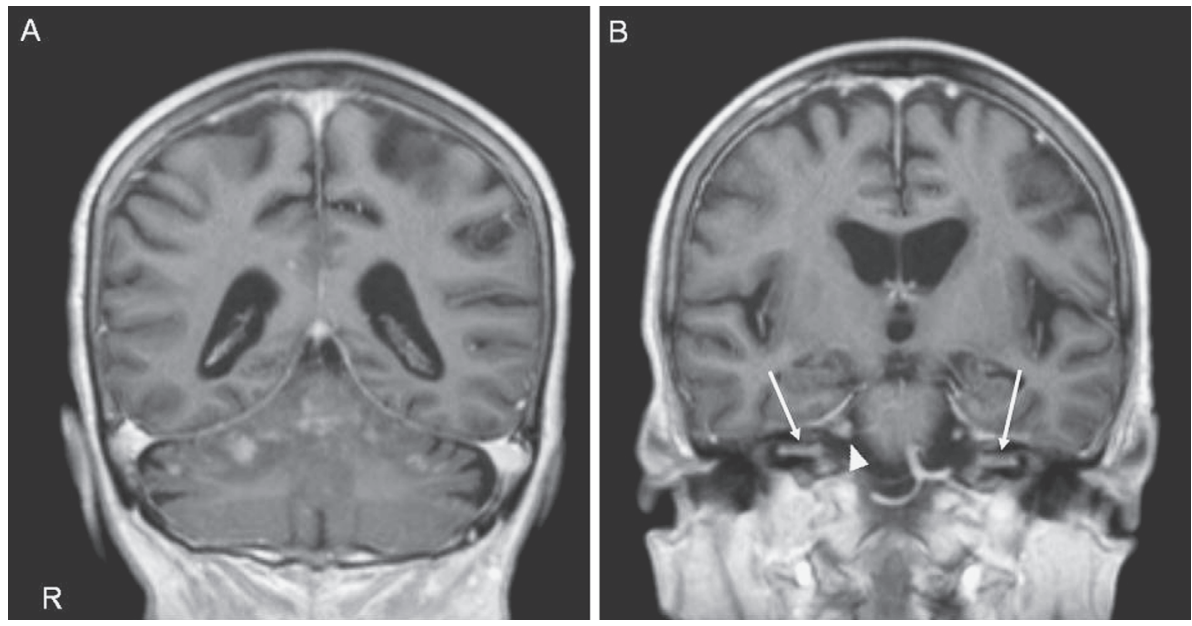

Figure 1. T1 coronal images after gadolinium administration showing enhancement along the surface of the cerebellum (A) and enhancement of the VII and VIII cranial nerves (arrows) in the internal acoustic meatus with a nodular enhancement on the right side (arrowhead) (B).

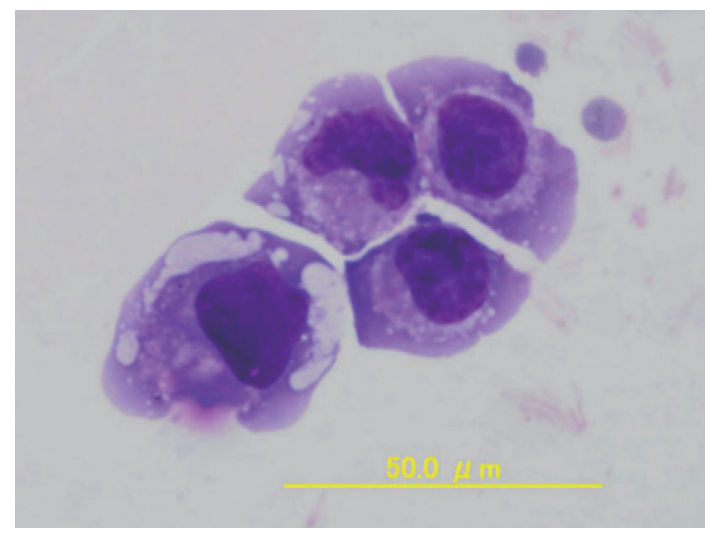

Figure 2. Cytology of the cerebrospinal fluid showed atypical cells with abnormally large and irregular shaped nuclei (Giemsa stain).

search for the primary tumor started. Her serum concentrations of carcinoembryogenic antigen (CEA) and carbohydrate antigen (CA) 19-9 were both increased: $141.3 \mathrm{ng} / \mathrm{mL}$ and $619.9 \mathrm{U} / \mathrm{mL}$, respectively. An abdominal CT scan revealed a thickened colon wall with narrowed lumen and multiple lymphadenopathies from the cervical to abdominal regions, without liver metastasis, indicating colon cancer (Fig. 3). Her level of consciousness continued to deteriorate significantly over the next two weeks and other symptoms appeared: extraocular palsy, masseter palsy, ileus and acute renal failure. She died from cachexia 6 weeks after admission to our hospital.

\section{Discussion}

The incidence of MC appears to be increasing, perhaps due to the longer survival of patients with malignancy. MC has been reported to occur in $2 \%$ to $25 \%$ of patients with malignancy $(1,2)$. Nevertheless, MC is still considered infrequent, and its variable clinical manifestations make clini-

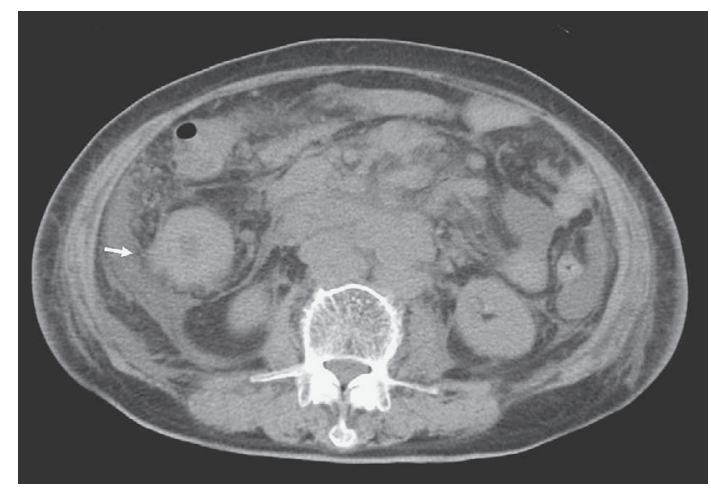

Figure 3. The abdominal CT scan revealed thickening of the wall of the ascending colon (arrow).

cal diagnosis difficult, especially in patients with unknown malignancies. The clinical manifestations of MC are heterogeneous and symptoms are typically widespread, involving multiple levels of the central and peripheral nervous system. The most frequently affected cranial nerves are III, IV, VI, VII (4). The case described here is atypical because the patient presented with progressive multiple cranial nerve palsy in the following order: VIII, VII, IX, X, III, IV, VI and V.

Bilateral deafness as the presenting symptom of $\mathrm{MC}$ is rare, and only eight cases with bilateral hearing loss as the first manifestation of a malignancy have been reported $(5,6)$. Moreover, no cases originating from the colon have been reported previously. The exact mechanism underlying the deafness is unclear. However, leptomeningeal spread within the confines of the internal auditory meati may cause deafness by direct compression of rigid surroundings. Vascular compromise may occur, as well as direct invasion and axonal destruction $(5,7)$. As with the present case, deafness precedes facial nerve paralysis in most cases (8). Sensory nerve fibers seem to be more vulnerable than motor nerve fibers (9).

The prognosis of MC is poor. The reported case was ad- 
mitted to our hospital 6 weeks before her death. Median survival in untreated patients is 4-6 weeks, which may be extended up to 6 months with aggressive treatment in some cases $(10,11)$. The poor prognosis and generally fast progression of MC make early diagnosis important.

In summary, we reported the rare case of MC in a patient who had bilateral deafness as the initial symptom of a previously undetected colon cancer malignancy. MC should be considered in the differential diagnosis in cases of abruptonset sensorineural deafness. Both MRI scans and CSF evaluation are necessary diagnostic tools, and should be used in conjunction as each of these procedures could have false-negative results. This applies even when there is no known primary malignancy.

\section{The authors state that they have no Conflict of Interest (COI).}

\section{References}

1. Wasserstrom WR, Glass JP, Posnar JB. Diagnosis and treatment of leptomeningeal metastasis from solid tumors: experience with 90 patients. Cancer 49: 759-772, 1982.
2. Grossman SA, Krabak MJ. Leptomeningeal carcinomatosis. Cancer Treat Rev 25: 103-119, 1999.

3. Kato H, Emura S, Takashima T, Ohmori K, Sunaga T. Gadolinium-enhanced magnetic resonance imaging of meningeal carcinomatosis in colon cancer. Tohoku J Exp Med 176: 121-126, 1995.

4. Gleissner B, Chamberlain MC. Neoplastic meningitis. Lancet Neurol 5: 443-452, 2006.

5. Civantos F, Choi YS, Applebaum EL. Meningeal carcinomatosis producing sudden hearing loss: A case report. Am J Otol 13: 369371, 1992.

6. Testoni S, Pirodda M, Trossello P, Minguzzi E, Alessandro RD. Meningeal carcinomatosis causing isolated bilateral symmetric progressive hearing loss. Neurol Sci 25: 345-347, 2005.

7. Oshiro H, Perlman HB. Subarachnoid spread of tumours in the labyrinth. Arch Otolaryngol Head Neck Surg 81: 328-334, 1965.

8. Imamura S, Nozawa I, Imamura M, Murakami Y. Clinicopathologic study of leptomeningeal carcinomatosis involving the temporal bone. Ann Otol Rhinol Laryngol 106: 674-679, 1997.

9. Igarashi M, Card GG, Johnson PE, Alford BR. Bilateral sudden hearing loss and metastatic adenocarcinoma. Arch Otolaryngol 105: 196-199, 1979.

10. Balm M, Hammack J. Leptomeningeal carcinomatosis. Presenting features and prognostic factors. Arch Neurol 53: 626-632, 1996.

11. Kim L, Glantz M. Neoplastic meningitis. Curr Treat Options Oncol 2: 517-527, 2001.

(C) 2012 The Japanese Society of Internal Medicine http://www.naika.or.jp/imindex.html 